\title{
Common fixed point theorems for two and three mappings
}

\author{
Meryeme El Harrak ${ }^{1 *}$ (D) and Ahmed Hajji ${ }^{1}$
}

\section{"Correspondence:}

elharrak57hollla@gmail.com

${ }^{1}$ LabMIA-SI, Department of

Mathematics, Faculty of Sciences,

Mohammed V University in Rabat,

Rabat, Morocco

\section{Springer}

\begin{abstract}
In this paper, we provide common fixed point theorems for two and three commuting mappings which generalize Darbo's fixed point theorem. An explicit example is given for illustration.
\end{abstract}

MSC: Primary 47H08; secondary 47H10

Keywords: Common fixed point; Measure of noncompactness; Darbo's fixed point theorem

\section{Introduction and preliminaries}

A contractive condition in terms of a measure of noncompactness, firstly used by Darbo [1], is one of the fruitful tools to obtain fixed point and common fixed point theorems. There are many extensions of this condition which are known as generalizations of Darbo's fixed point theorem; see for example [2-11] and the references therein.

In recent papers, Hajji-Hanebaly [6] and Hajji [7] proved some fixed point theorems for a pair of commuting operators, generalizing Darbo's, Sadovski's and Markov-Kakutani's fixed point theorems. In [8], Khodabakhshi obtained some new common fixed point results using the technique associated with a measure of noncompactness for two commuting operators.

In the present paper, we make use of some properties of measure of noncompactness to establish new contraction conditions giving rise to common fixed point theorems for two and three commuting mappings. The obtained results can be seen as specific generalizations of Darbo's fixed point theorem. For illustration, we provide in Sect. 3 a concrete example for which both the classical Darbo's theorem and its generalization due to Hajji [7] are not applicable. This example shows the powerfulness of the obtained results.

To this end, we begin by fixing some notations, definitions and auxiliary facts which will be needed in the sequel. Let $X$ be a given Banach space. If $B$ is a subset of $X$ then the symbols $\bar{B}$ and $\operatorname{conv}(B)$ stand for the closure and the convex hull of $B$, respectively. Moreover, we denote by $\mathfrak{M}_{X}$ the family of all nonempty and bounded subsets of $X$ and by $\mathfrak{N}_{X}$ its subfamily consisting of all relatively compact sets.

From [12], we recall the definition of the measure of noncompactness.

(c) The Author(s) 2020. This article is licensed under a Creative Commons Attribution 4.0 International License, which permits use sharing, adaptation, distribution and reproduction in any medium or format, as long as you give appropriate credit to the original author(s) and the source, provide a link to the Creative Commons licence, and indicate if changes were made. The images or other third party material in this article are included in the article's Creative Commons licence, unless indicated otherwise in a credit line to the material. If material is not included in the article's Creative Commons licence and your intended use is not permitted by statutory regulation or exceeds the permitted use, you will need to obtain permission directly from the copyright holder. To view a copy of this licence, visit http://creativecommons.org/licenses/by/4.0/. 
Definition 1.1 A mapping $\mu: \mathfrak{M}_{X} \rightarrow[0, \infty)$ is said to be a measure of noncompactness in $X$ if it satisfies the following conditions:

(i) The family $\operatorname{ker} \mu=\left\{A \in \mathfrak{M}_{X}: \mu(A)=0\right\}$ is nonempty and ker $\mu \subseteq \mathfrak{N}_{X}$.

(ii) $A \subseteq B \Rightarrow \mu(A) \leq \mu(B)$.

(iii) $\mu(\bar{A})=\mu(A)$.

(iv) $\mu(\operatorname{conv}(A))=\mu(A)$.

(v) $\mu(\lambda A+(1-\lambda) B) \leq \lambda \mu(A)+(1-\lambda) \mu(B)$, for any $\lambda \in[0,1]$.

(vi) If $\left(A_{n}\right)_{n}$ is a sequence of closed sets from $\mathfrak{M}_{X}$ such that $A_{n+1} \subseteq A_{n}$ for $n=1,2, \ldots$, and if $\lim _{n \rightarrow+\infty} \mu\left(A_{n}\right)=0$, then the set $A_{\infty}=\bigcap_{n=1}^{\infty} A_{n}$ is nonempty.

The family ker $\mu$ defined in axiom (i) is called the kernel of the measure of noncompactness.

One of the well-known fixed point theorems is due to Schauder. It can be stated as follows [13].

Theorem 1.2 (Schauder's fixed point theorem) Let $\Omega$ be a nonempty, compact and convex subset of a Banach space X. Then each continuous map $T: \Omega \rightarrow \Omega$ has at least one fixed point in $\Omega$.

As a generalization of the above result, we have Darbo's fixed point theorem [12].

Theorem 1.3 (Darbo's fixed point theorem) Let $\Omega$ be a nonempty, bounded, closed and convex subset of a Banach space $X$ and let $T: \Omega \rightarrow \Omega$ be a continuous mapping. Assume that there exists a constant $k \in[0,1)$ such that

$$
\mu(T A) \leq k \mu(A)
$$

for any subset $A$ of $\Omega$. Then $T$ has a fixed point in $\Omega$.

Definition 1.4 ([14]) A mapping $T$ on a convex set $A$ is said to be affine if it satisfies the identity

$$
T(k x+(1-k) y)=k T x+(1-k) T y,
$$

whenever $0<k<1$, and $x, y \in A$.

Recently, Hajji obtained in [7] a common fixed point theorem for commuting operators, generalizing Darbo's fixed point theorem. Namely, we assert the following.

Theorem 1.5 ([7]) Let $\Omega$ be a nonempty, bounded, closed and convex subset of a Banach space $X$ and let $T$ and $S$ be two continuous mappings from $\Omega$ into $\Omega$ such that

(a) $T S=S T$.

(b) $T$ is affine.

(c) There exists $k \in[0,1)$ such that, for any $A \subseteq \Omega$, we have

$$
\mu(T S(A)) \leq k \mu(A) .
$$

Then the set $\{x \in \Omega: T x=S x=x\}$ is nonempty and compact. 


\section{Main results}

In this section, we state and prove our main results on common fixed point theorems for two commuting mappings. We also extend the obtained results to the case of three mappings.

Theorem 2.1 Let $\Omega$ be a nonempty, bounded, closed and convex subset of a Banach space $X$ and let $T$ and $S$ be two continuous mappings from $\Omega$ into $\Omega$ such that

(a) $T S=S T$.

(b) For any nonempty subset $A$ of $\Omega$, we have

$$
\sigma(A) \leq \varphi(S A)-\varphi(S(\overline{\operatorname{conv}}(T A)))
$$

where $\sigma, \varphi: P(\Omega) \rightarrow[0,+\infty)$ are mappings such that $\sigma$ satisfies axioms (i), (ii) and (vi) in Definition 1.1.

Then

(1) $T$ has at least one fixed point in $\Omega$.

(2) If for any nonempty subset $A$ of $\Omega$ we have $S(\overline{\operatorname{conv}}(A)) \subseteq \overline{\operatorname{conv}}(S(A))$, then $S$ has at least one fixed point in $\Omega$.

(3) If $S$ is affine, then $S$ and $T$ have a common fixed point in $\Omega$.

Remark 2.2 We recover Darbo's fixed point theorem by specifying $S, \sigma$ and $\varphi$, for instance, by letting $S$ be the identity mapping, and taking $\sigma=\mu$ and $\varphi=\left(\frac{1}{1-k}\right) \mu, \mu$ being a measure of noncompactness and $k \in[0,1)$.

Proof (1) We define the sequence $\left\{\Omega_{n}\right\}$ as follows:

$$
\left\{\begin{array}{l}
\Omega_{0}=\Omega, \\
\Omega_{n}=\overline{\operatorname{conv}}\left(T \Omega_{n-1}\right), \quad n=1,2, \ldots
\end{array}\right.
$$

For $n=1$, we have $\Omega_{1} \subseteq \Omega_{0}$. Suppose that $\Omega_{n} \subseteq \Omega_{n-1}$ is true for some $n \geq 1$. Then

$$
\Omega_{n+1}=\overline{\operatorname{conv}}\left(T\left(\Omega_{n}\right)\right) \subseteq \overline{\operatorname{conv}}\left(T\left(\Omega_{n-1}\right)\right)=\Omega_{n} .
$$

By induction, we get $\Omega_{n} \subseteq \Omega_{n-1}$ for every $n \geq 1$.

Now, let us consider $a_{n}=\varphi\left(S \Omega_{n}\right)$. From inequality (2.1), we have

$$
\varphi\left(S \Omega_{n}\right)-\varphi\left(S\left(\overline{\operatorname{conv}}\left(T \Omega_{n}\right)\right)\right) \geq 0, \quad \text { for all } n \in \mathbb{N} \cup\{0\} .
$$

It follows that

$$
a_{n+1}=\varphi\left(S \Omega_{n+1}\right)=\varphi\left(S\left(\overline{\operatorname{conv}}\left(T \Omega_{n}\right)\right)\right) \leq \varphi\left(S \Omega_{n}\right)=a_{n}, \quad \text { for all } n \in \mathbb{N} \cup\{0\} .
$$

Hence, $\left\{a_{n}\right\}$ is a positive non-increasing sequence of real numbers, and therefore there exists $a \geq 0$ such that $a_{n} \rightarrow a$ as $n \rightarrow \infty$. Using inequality (2.1) again, we obtain

$$
\sigma\left(\Omega_{n}\right) \leq \varphi\left(S \Omega_{n}\right)-\varphi\left(S\left(\overline{\operatorname{conv}}\left(T \Omega_{n}\right)\right)\right)=a_{n}-a_{n+1},
$$


which implies that

$$
\lim _{n \rightarrow+\infty} \sigma\left(\Omega_{n}\right)=0
$$

From (vi) of Definition 1.1, we deduce that the set $\Omega_{\infty}=\bigcap_{n=1}^{\infty} \Omega_{n}$ is nonempty. The fact that $\Omega_{\infty} \subseteq \Omega_{n}$, for every $n \in \mathbb{N}$, as well as (ii) of Definition 1.1 infer

$$
\sigma\left(\Omega_{\infty}\right) \leq \sigma\left(\Omega_{n}\right), \quad \text { for all } n \in \mathbb{N} \text {. }
$$

Passing to the limit, we get $\sigma\left(\Omega_{\infty}\right)=0$. Thus, by using (i) of Definition 1.1, we conclude that $\overline{\Omega_{\infty}}=\Omega_{\infty}$ is compact. Moreover, $\Omega_{\infty}$ is convex since each $\Omega_{n}$ is convex. Note also that

$$
T\left(\Omega_{n}\right) \subseteq \overline{\operatorname{conv}}\left(T \Omega_{n}\right)=\Omega_{n+1} \subseteq \Omega_{n}, \quad n=0,1,2, \ldots
$$

Then $T\left(\Omega_{\infty}\right) \subseteq \Omega_{\infty}$. By Theorem 1.2, $T$ has a fixed point in $\Omega$.

(2) Here we use Theorem 1.2 again. From part (1), we have $\Omega_{\infty}$ is nonempty, compact and convex. So it remains to prove $S\left(\Omega_{\infty}\right) \subseteq \Omega_{\infty}$. To do this, it suffices to show that $S\left(\Omega_{n}\right) \subseteq \Omega_{n}$ for every $n=1,2, \ldots$

For $n=1$, we have

$$
S\left(\Omega_{1}\right)=S\left(\overline{\operatorname{conv}}\left(T \Omega_{0}\right)\right) \subseteq \overline{\operatorname{conv}}\left(S\left(T \Omega_{0}\right)\right)=\overline{\operatorname{conv}}\left(T\left(S \Omega_{0}\right)\right) \subseteq \Omega_{1}
$$

Assuming now that $S\left(\Omega_{n}\right) \subseteq \Omega_{n}$ is true for some $n \geq 1$. Then

$$
S\left(\Omega_{n+1}\right)=S\left(\overline{\operatorname{conv}}\left(T \Omega_{n}\right)\right) \subseteq \overline{\operatorname{conv}}\left(S\left(T \Omega_{n}\right)\right) \subseteq \Omega_{n+1} .
$$

By induction, we get $S\left(\Omega_{n}\right) \subseteq \Omega_{n}$ for every $n \geq 1$. We can deduce that $S\left(\Omega_{\infty}\right) \subseteq \Omega_{\infty}$. By Theorem 1.2, $S$ has a fixed point in $\Omega$.

(3) The set $F=\{x \in \Omega: S(x)=x\}$ is bounded since $F \subseteq \Omega$ and $\Omega$ is bounded, closed by the continuity of $S$ and convex since $S$ is affine. On the other hand, we can see that $S(F) \subseteq F$ and by commutativity of the operators $T$ and $S$, we get

$$
S(T(x))=T(S(x))=T(x) .
$$

This to say that $T(x)$ is a fixed point of $S$ for any $x \in F$. Thus $T(F) \subseteq F$ and

$$
\sigma(A) \leq \varphi(S A)-\varphi(S(\overline{\operatorname{conv}}(T A))), \quad \text { for every } A \subseteq F .
$$

Then by part (1), $T$ has a fixed point in $F$ and therefore $S$ and $T$ have a common fixed point.

Theorem 2.3 Let $\Omega$ be a nonempty, bounded, closed and convex subset of a Banach space $X$ and let $T$ and $S$ be two continuous mappings from $\Omega$ into $\Omega$ such that

(a) $S$ is affine.

(b) $T S=S T$. 
(c) For any nonempty subset $A$ of $\Omega$, we have

$$
\sigma(S A) \leq \varphi(A)-\varphi(\overline{\operatorname{conv}}(T A))
$$

where $\sigma, \varphi: P(\Omega) \rightarrow[0,+\infty)$ are mappings such that $\sigma$ satisfies axioms (i), (ii), (iii) and (vi) in Definition 1.1.

\section{Then}

(1) $T$ and $S$ have a fixed point in $\Omega$.

(2) $T$ and $S$ have a common fixed point in $\Omega$.

Proof (1) We proceed in a similar way to the proof of (1) in the previous theorem. Thus, for given sequence $\left\{\Omega_{n}\right\}$ as in the proof of Theorem 2.1, inequality (2.2) yields

$$
\varphi\left(\Omega_{n}\right)-\varphi\left(\overline{\operatorname{conv}}\left(T \Omega_{n}\right)\right) \geq 0, \quad \text { for all } n \in \mathbb{N} \cup\{0\} .
$$

Let $b_{n}=\varphi\left(\Omega_{n}\right)$, we obtain

$$
b_{n+1}=\varphi\left(\Omega_{n+1}\right)=\varphi\left(\overline{\operatorname{conv}}\left(T \Omega_{n}\right)\right) \leq \varphi\left(\Omega_{n}\right)=b_{n}, \quad \text { for all } n \in \mathbb{N} \cup\{0\}
$$

Therefore, $\left\{b_{n}\right\}$ is a positive non-increasing sequence of real numbers, then there exists $b \geq 0$ such that $b_{n} \rightarrow b$ as $n \rightarrow \infty$. Moreover, from inequality (2.2), we have

$$
\sigma\left(S \Omega_{n}\right) \leq \varphi\left(\Omega_{n}\right)-\varphi\left(\overline{\operatorname{conv}}\left(T \Omega_{n}\right)\right)=b_{n}-b_{n+1} .
$$

This implies that $\lim _{n \rightarrow+\infty} \sigma\left(S \Omega_{n}\right)=0$. Define $\Omega_{n}^{\prime}=\overline{\mathrm{S}\left(\Omega_{n}\right)}$, from (iii) of Definition 1.1, we get

$$
\sigma\left(\Omega_{n}^{\prime}\right)=\sigma\left(\overline{\mathrm{S}\left(\Omega_{n}\right)}\right)=\sigma\left(S\left(\Omega_{n}\right)\right)
$$

so $\lim _{n \rightarrow+\infty} \sigma\left(\Omega_{n}^{\prime}\right)=0$. Since the sequence $\left\{\Omega_{n}\right\}$ is nested, we have $\Omega_{n+1}^{\prime} \subseteq \Omega_{n}^{\prime}$ for all $n \in \mathbb{N}$. In view of property (vi) of Definition $1.1, \Omega_{\infty}^{\prime}=\bigcap_{n=1}^{\infty} \Omega_{n}^{\prime}$ is nonempty. As $\Omega_{\infty}^{\prime} \subseteq \Omega_{n}^{\prime}$, for every $n \in \mathbb{N}$, from (ii) of Definition 1.1, we obtain

$$
\sigma\left(\Omega_{\infty}^{\prime}\right) \leq \sigma\left(\Omega_{n}^{\prime}\right) \text { for all } n \in \mathbb{N} \text {. }
$$

As $n$ tends t*o infinity, we obtain $\sigma\left(\Omega_{\infty}^{\prime}\right)=0$. It follows that $\overline{\Omega_{\infty}^{\prime}}=\Omega_{\infty}^{\prime}$ is compact by means of (i) of Definition 1.1. Moreover, $\Omega_{\infty}^{\prime}$ is convex since $S$ is affine. On the other hand, we have $T\left(\Omega_{n}\right) \subseteq \Omega_{n}$ and $S\left(\Omega_{n}\right) \subseteq \Omega_{n}$ which were proved in Theorem 2.1. So we get

$$
T\left(\Omega_{n}^{\prime}\right) \subseteq T\left(\overline{S\left(\Omega_{n}\right)}\right) \subseteq \overline{T\left(S\left(\Omega_{n}\right)\right)} \subseteq \overline{S\left(T\left(\Omega_{n}\right)\right)} \subseteq \overline{S\left(\Omega_{n}\right)}=\Omega_{n}^{\prime}
$$

and

$$
S\left(\Omega_{n}^{\prime}\right) \subseteq S\left(\overline{S\left(\Omega_{n}\right)}\right) \subseteq S\left(\overline{\Omega_{n}}\right) \subseteq \overline{S\left(\Omega_{n}\right)}=\Omega_{n}^{\prime}, \quad n=0,1,2, \ldots
$$

This shows that the set $\Omega_{\infty}^{\prime}$ is invariant under the operators $T$ and $S$. Thus, applying Theorem 1.2, $S$ and $T$ have a fixed point in $\Omega$. 
(2) In the same way as in part (3) of the proof of Theorem 2.1, we see that $F=\{x \in \Omega$ : $S(x)=x\}$ is convex, closed and bounded subset of $\Omega, T(F) \subseteq F$ and $S(F) \subseteq F$. In addition, from inequality 2.2 , we have

$$
\sigma(S A) \leq \varphi(A)-\varphi(\overline{\operatorname{conv}}(T A)), \quad \text { for every } A \subseteq F
$$

Part (1) shows that $T$ has a fixed point in $F$ and therefore $S$ and $T$ have a common fixed point in $\Omega$.

Now, motivated by the last two results, we establish the following common fixed point theorem for three mappings.

Theorem 2.4 Let $\Omega$ be a nonempty, bounded, closed and convex subset of a Banach space $X$ and let $T, S$ and $H$ be three continuous mappings from $\Omega$ into $\Omega$ such that

(a) $H$ and $S$ are affine.

(b) $T S=S T, T H=H T, S H=H S$.

(c) For any nonempty subset $A$ of $\Omega$, we have

$$
\sigma(H A) \leq \varphi(S A)-\varphi(S(\overline{\operatorname{conv}}(T A)))
$$

where $\sigma, \varphi: P(\Omega) \rightarrow[0,+\infty)$ are mappings such that $\sigma$ satisfies axioms (i), (ii), (iii) and (vi) in Definition 1.1.

Then

(1) $T, S$ and $H$ have a fixed point in $\Omega$.

(2) $T, S$ and $H$ have a common fixed point in $\Omega$.

Proof (1) Again, we consider the sequence $\left\{\Omega_{n}\right\}$ defined in the proof of Theorem 2.1 and let $c_{n}=\varphi\left(S \Omega_{n}\right)$. From inequality (2.3), we have

$$
\varphi\left(S \Omega_{n}\right)-\varphi\left(S\left(\overline{\operatorname{conv}}\left(T \Omega_{n}\right)\right) \geq 0, \quad \text { for all } n \in \mathbb{N} \cup\{0\}\right.
$$

This implies that

$$
c_{n+1}=\varphi\left(S \Omega_{n+1}\right)=\varphi\left(S\left(\overline{\operatorname{conv}}\left(T \Omega_{n}\right)\right)\right) \leq \varphi\left(S \Omega_{n}\right)=c_{n}, \quad \text { for all } n \in \mathbb{N} \cup\{0\}
$$

Therefore, $\left\{c_{n}\right\}$ is a positive non-increasing sequence of real numbers, then there exists $c \geq 0$ such that $c_{n} \rightarrow c$ as $n \rightarrow \infty$. Also, from inequality (2.3), we have

$$
\sigma\left(H \Omega_{n}\right) \leq \varphi\left(S \Omega_{n}\right)-\varphi\left(S\left(\overline{\operatorname{conv}}\left(T \Omega_{n}\right)\right)\right)=c_{n}-c_{n+1},
$$

so $\lim _{n \rightarrow+\infty} \sigma\left(H \Omega_{n}\right)=0$. Now, if we set $\Omega_{n}^{\prime \prime}=\overline{\mathrm{H}\left(\Omega_{n}\right)}$, we can make use of (iii) of Definition 1.1 , to show

$$
\sigma\left(\Omega_{n}^{\prime \prime}\right)=\sigma\left(\overline{H\left(\Omega_{n}\right)}\right)=\sigma\left(H\left(\Omega_{n}\right)\right)
$$

so that one can conclude $\lim _{n \rightarrow+\infty} \sigma\left(\Omega_{n}^{\prime \prime}\right)=0$. Since the sequence $\left\{\Omega_{n}\right\}$ is nested, we have $\Omega_{n+1}^{\prime \prime} \subseteq \Omega_{n}^{\prime \prime}$ for all $n \in \mathbb{N}$. Therefore, by the property (vi) of Definition $1.1 \Omega_{\infty}^{\prime \prime}=\bigcap_{n=1}^{\infty} \Omega_{n}^{\prime \prime}$ 
is nonempty. Moreover, by using (ii) of Definition 1.1, we get

$$
\sigma\left(\Omega_{\infty}^{\prime \prime}\right) \leq \sigma\left(\Omega_{n}^{\prime \prime}\right) \text { for all } n \in \mathbb{N}
$$

Therefore, $\sigma\left(\Omega_{\infty}^{\prime \prime}\right)=0$, with $n$ tending to infinity. This when combined with (i) of Definition 1.1 give rise to that $\overline{\Omega_{\infty}^{\prime \prime}}=\Omega_{\infty}^{\prime \prime}$ is compact and convex since $H$ is affine. On the other hand, making use of $T\left(\Omega_{n}\right) \subseteq \Omega_{n}$ and $S\left(\Omega_{n}\right) \subseteq \Omega_{n}$ (see Theorem 2.1), we obtain

$$
T\left(\Omega_{n}^{\prime \prime}\right)=T\left(\overline{H\left(\Omega_{n}\right)}\right) \subseteq \overline{T\left(H\left(\Omega_{n}\right)\right)} \subseteq \overline{H\left(T\left(\Omega_{n}\right)\right)} \subseteq \overline{H\left(\Omega_{n}\right)}=\Omega_{n}^{\prime \prime}
$$

and

$$
\left.S\left(\Omega_{n}^{\prime \prime}\right)=S\left(\overline{H\left(\Omega_{n}\right)}\right) \subseteq \overline{H\left(S\left(\Omega_{n}\right)\right.}\right) \subseteq \overline{H\left(\Omega_{n}\right)}=\Omega_{n}^{\prime \prime}, \quad n=0,1,2, \ldots
$$

Similarly to $S$, in the proof of Theorem 2.1, we can prove $H\left(\Omega_{n}\right) \subseteq \Omega_{n}$. So

$$
\left.H\left(\Omega_{n}^{\prime \prime}\right)=H\left(\overline{H\left(\Omega_{n}\right)}\right) \subseteq \overline{H\left(H\left(\Omega_{n}\right)\right.}\right) \subseteq \overline{H\left(\Omega_{n}\right)}=\Omega_{n}^{\prime \prime}, \quad n=0,1,2, \ldots
$$

Hence, the set $\Omega_{\infty}^{\prime \prime}$ is invariant under the operators $T, S$ and $H$. Thus, Theorem 1.2 implies that $S, T$ and $H$ have a fixed point.

(2) By the same argument as in part (3) of the proof of Theorem 2.1, we see that $F=$ $\{x \in \Omega: H(x)=x\}$ is convex, closed and bounded subset of $\Omega, S(F) \subseteq F$ and $H(F) \subseteq F$. Moreover, from inequality 2.4 , we have

$$
\sigma(H A) \leq \varphi(S A)-\varphi(S(\overline{\operatorname{conv}}(T A))), \quad \text { for every } A \subseteq F
$$

Then by part (1), the mapping $S$ has a fixed point in $F$ and therefore $S$ and $H$ have a common fixed point. In a similar way, we can prove that $T$ has a fixed point in $K=\{x \in \Omega$ : $S(x)=H(x)=x\}$. Therefore $S, H$ and $T$ have a common fixed point.

An immediate consequence of the previous theorem is the following.

Corollary 2.5 Let $\Omega$ be a nonempty, bounded, closed and convex subset of a Banach space $X$ and let $T, S$ and $H$ be continuous mappings from $\Omega$ into $\Omega$ such that

(a) $H$ and $S$ are affine.

(b) $T S=S T, T H=H T, S H=H S$.

(c) For all $x, y \in \Omega$

$$
d(H x, H y) \leq \beta(S x, S y)-\beta(S T x, S T y),
$$

where $d$ is a metric on $X$ and $\beta: \Omega \times \Omega \rightarrow \mathbb{R}^{+}$is a mapping. Then the set $\{x \in \Omega: T x=$ $S x=H x=x\}$ is nonempty.

Proof Let $\mu: \mathfrak{M}_{X} \rightarrow[0, \infty)$ be the mapping defined by the formula

$$
\sigma(A)=\operatorname{diam}(A)
$$


where $\operatorname{diam}(A)=\sup \{d(x, y): x, y \in A\}$ stands for the diameter of $A$. It is easily seen that $\sigma$ is a measure of noncompactness in a space $X$. In view of (2.4), we have

$$
\inf _{x, y \in A} \beta(S T x, S T y) \leq \inf _{x, y \in A} \beta(S x, S y)-\sup _{x, y \in A} d(H x, H y) .
$$

This yields

$$
\sup _{x, y \in A} d(H x, H y) \leq \inf _{x, y \in A} \beta(S x, S y)-\inf _{x, y \in A} \beta(S T x, S T y) .
$$

Set $\varphi(A)=\inf _{x, y \in A} \beta(x, y)$, we obtain

$$
\operatorname{diam}(H(A)) \leq \varphi(S(A))-\varphi(S T(A))
$$

Since $S T(A) \subseteq S(\overline{\operatorname{conv}}(T(A)))$, we get

$$
\varphi(S(\overline{\operatorname{conv}}(T A))) \leq \varphi(S T(A)) .
$$

So

$$
\sigma(H A) \leq \varphi(S A)-\varphi(S(\overline{\operatorname{conv}}(T A)))
$$

The application of Theorem 2.4 completes the proof.

To support our results, we give an illustrative example. Precisely, we show that Corollary 2.5 can be used to cover this example while the results due to Darbo [1] and Hajji [7] are not applicable.

\section{Example}

Let $X=L^{1}\left(\mathbb{R}_{+}\right)$be the space of Lebesgue integrable functions on the measurable subset $\mathbb{R}_{+}$of $\mathbb{R}$ with the standard norm

$$
\|x\|=\int_{0}^{+\infty}|x(t)| d t
$$

Let $k \in L^{1}\left(\mathbb{R}_{+}\right)$defined in [7] by

$$
k(t)= \begin{cases}e^{-t}, & t \in[0,1], \\ 0, & t>1 .\end{cases}
$$

For any $A>0$ and for all $t_{1}, t_{2} \in \mathbb{R}_{+}$, the following condition is satisfied (see [7]):

$$
t_{1}<t_{2} \quad \Longrightarrow \quad \int_{0}^{A} k\left(t_{2}-s\right) d s \leq \int_{0}^{A} k\left(t_{1}-s\right) d s
$$

Then, using Krzyz's theorem (see [15]), the linear integral operator

$$
(K x)(t)=\int_{0}^{\infty} k(t-s) x(s) d s
$$


transforms the set of non-increasing functions from $L^{1}\left(\mathbb{R}_{+}\right)$into itself. Furthermore, the norm of the convolution operator $K$ satisfies (see [7])

$$
\|K\| \leq 1-e^{-1}
$$

Now, let us consider the three operators defined by

$$
\begin{aligned}
& T x(t)=2 e^{-1}\left(1-e^{-1}\right) x(t)+e^{-t}, \\
& S x(t)=\frac{e}{e-1} K x(t), \\
& H x(t)=\frac{B}{\left(1-e^{-1}\right)^{3}} K^{3}(x(\phi(t))),
\end{aligned}
$$

for any $x \in L^{1}\left(\mathbb{R}_{+}\right)$, where $b>0$, the function $\phi: \mathbb{R}_{+} \rightarrow \mathbb{R}_{+}$is increasing and absolutely continuous such that $\phi^{\prime}(t) \geq B$ for some constant $B>0$ and for almost all $t \in \mathbb{R}_{+}$and $\frac{b}{B}(1-$ $\left.e^{-1}\right)^{3} \leq 1$. For any $x \in L^{1}\left(\mathbb{R}_{+}\right)$, we have

$$
\|T x\| \leq \int_{0}^{\infty} e^{-t} d t+2 e^{-1}\left(1-e^{-1}\right)\|x\| \leq 1+2 e^{-1}\left(1-e^{-1}\right)\|x\| .
$$

Hence, for $\|x\| \leq r$, we have

$$
\|T x\| \leq 1+2 e^{-1}\left(1-e^{-1}\right) r
$$

and if we take

$$
1+2 e^{-1}\left(1-e^{-1}\right) r=r
$$

then

$$
r=\frac{1}{1-2 e^{-1}\left(1-e^{-1}\right)} .
$$

Moreover, for $\|x\| \leq r$, we have

$$
\|S x\| \leq \frac{e}{e-1}\|K\|\|x\| \leq \frac{1}{1-e^{-1}}\left(1-e^{-1}\right)\|x\|=\|x\| \leq r
$$

and

$$
\|H x\| \leq \frac{b\|K\|^{3}}{B}\|x\| \leq \frac{b}{B}\left(1-e^{-1}\right)^{3}\|x\| \leq\|x\| \leq r .
$$

This implies that $T, S$ and $H$ map $B_{r}$ into $B_{r}$, where $r=\frac{1}{1-2 e^{-1}\left(1-e^{-1}\right)}$. Further, let $\Omega=Q_{r}$ be the subset of the ball $B_{r}$ consisting of all functions that are a.e positive and non-increasing on $\mathbb{R}_{+}$, which is nonempty, bounded, closed and convex subset of $L^{1}\left(\mathbb{R}_{+}\right)$(see [16]). Thus, since $T, S$ and $H$ are positive and non-increasing on $\mathbb{R}_{+}, T, S$ and $H$ map $Q_{r}$ into $Q_{r}$. Whence, for any $A \subseteq Q_{r}$, we have

$$
\sigma(S A) \leq \sigma(A)
$$




$$
\begin{aligned}
& \sigma(H A) \leq \sigma(A), \\
& \sigma(H S A) \leq \sigma(A),
\end{aligned}
$$

where $\sigma(A)=\sup \{\|x-y\|: x, y \in A\}$. Observe that $S, H$ and $S H$ are $\sigma$-non-expansive. Then neither Darbo's fixed point theorem [1] nor Hajji's fixed point theorem [7] can be applied. On the other hand, for $x, y \in Q_{r}$, we have

$$
\begin{aligned}
\|H x-H y\| & \leq \frac{b}{B}\left(1-e^{-1}\right)^{2}\|K x-K y\| \\
& \leq \frac{b}{B}\left[\left(1+e^{-2}\right)\|K x-K y\|-2 e^{-1}\|K x-K y\|\right] \\
& \leq \frac{b}{B}\left[\frac{e}{e-1}\|K x-K y\|-2 e^{-1}\|K x-K y\|\right] \\
& \leq \frac{b}{B}\left[\|S x-S y\|-2 e^{-1} \frac{e-1}{e} \frac{e}{e-1}\|K x-K y\|\right] \\
& \leq \frac{b}{B}\left[\|S x-S y\|-2 e^{-1}\left(1-e^{-1}\right)\|S x-S y\|\right] \\
& \leq \frac{b}{B}\|S x-S y\|-\frac{b}{B}\left\|2 e^{-1}\left(1-e^{-1}\right)(S x-S y)\right\| .
\end{aligned}
$$

Therefore

$$
\|H x-H y\| \leq \frac{b}{B}\|S x-S y\|-\frac{b}{B}\|T S x-T S y\| .
$$

Taking $\beta(x, y)=\frac{b}{B}\|x-y\|$, we get

$$
d(H x, H y)=\|H x-H y\| \leq \beta(S x, S y)-\beta(S T x, S T y) .
$$

Moreover, $S$ and $H$ commute and, since the function $e^{-t}$ for any $t \in \mathbb{R}_{+}$is a fixed point of $K$, we have $T S=S T$ and $T H=H T$ (see [7]). Finally, since $S$ and $H$ are affine, we conclude from Corollary 2.4 that $T, S$ and $H$ have a common fixed point $L^{1}\left(\mathbb{R}_{+}\right)$.

\section{Acknowledgements}

The authors would like to thank the reviewers and the editor for their valuable comments.

Funding

Not applicable.

\section{Availability of data and materials}

Not applicable.

\section{Competing interests}

The authors declare that they have no competing interests.

Authors' contributions

All authors contributed equally. All authors read and approved the final manuscript.

\section{Publisher's Note}

Springer Nature remains neutral with regard to jurisdictional claims in published maps and institutional affiliations.

Received: 18 July 2019 Accepted: 2 July 2020 Published online: 20 July 2020 
References

1. Darbo, G.: Punti uniti in transformazioni a condominio non compatto (in Italy). Rend. Semin. Mat. Univ. Padova 2, 84-92 (1955)

2. Aghajani, A., Aliaskari, M.: Generalization of Darbo's fixed point theorem and application. Int. J. Nonlinear Anal. Appl. 2(2), 86-95 (2011)

3. Aghajani, A., Banaś, J., Sabzali, N.: Some generalizations of Darbo fixed point theorem and applications. Bull. Belg. Math. Soc. Simon Stevin 20(2), 345-358 (2013)

4. Arab, R.: Some generalizations of Darbo fixed point theorem and its application. Miskolc Math. Notes 18(2), 595-610 (2017)

5. Cai, L., Liang, L.: New generalizations of Darbo's fixed point theorem. Fixed Point Theory Appl. 2015(1), 1 (2015)

6. Hajji, A., Hanebaly, E.: Commuting mappings and $\alpha$-compact type fixed point theorems in locally convex spaces. Int. J. Math. Anal. 1(14), 661-680 (2007)

7. Hajji, A.: A generalization of Darbo's fixed point and common solutions of equations in Banach spaces. Fixed Point Theory Appl. 2013(1), 62 (2013)

8. Khodabakhshi, N., Vaezpour, S.M.: Common fixed point theorems via measure of noncompactness. Fixed Point Theory 17(2), 381-386 (2016)

9. Nikbakhtsarvestani, F., Vaezpour, S.M., Asadi, M.: $f(\psi, \varphi)$-contraction in terms of measure of noncompactness with application for nonlinear integral equations. J. Inequal. Appl. 2017(1), 271 (2017)

10. Samadi, A., Ghaemi, M.B.: An extension of Darbo's theorem and its application. In: Abstr. Appl. Anal. Hindawi (2014)

11. Sarvestani, F.N., Vaezpour, S.M., Asadi, M.: Some generalization of Darbo's fixed point. J. Math. Anal. 7(3), 78-94 (2016)

12. Banas, J., Goebel, K.: Measures of noncompactness in Banach spaces. Lect. Notes Pure Appl. Math 60 (1980)

13. Agarwal, R.P., Meehan, M., O'regan, D.: Fixed Point Theory and Applications. Cambridge university press, Cambridge (2004)

14. Dugungii, J., Granas, A.: Fixed Point Theory I. Polish Sci. Publ., Warszawa (1998)

15. Krzyz, J.: On monotonicity-preserving transformations. Ann. Univ. Mariae Curie-Skłodowska, Sect. A 6, $91-111$ (1952)

16. El-Sayed, W.G.: Nonlinear functional integral equations of convolution type. Port. Math. 54(4), 449-456 (1997)

\section{Submit your manuscript to a SpringerOpen ${ }^{\circ}$ journal and benefit from:}

- Convenient online submission

- Rigorous peer review

Open access: articles freely available online

- High visibility within the field

- Retaining the copyright to your article

Submit your next manuscript at $\gg$ springeropen.com 